\title{
New supernova constraints on active-sterile neutrino conversions
}

\author{
S. Pastor, V.B. Semikoz ${ }^{1}$, J.W.F. Valle ${ }^{2}$ \\ Instituto de Física Corpuscular - C.S.I.C., Departament de Física Tè̀rica, Universitat de València, 46100 Burjassot, València, Spain
}

Received 2 August 1994

\begin{abstract}
We consider active-sterile neutrino conversions in a supernova in the presence of random magnetic field domains. For large enough fields the magnetization of the medium may enhance the active to sterile neutrino conversion rates. Neglecting neutrino transition magnetic moments we show that for $\mathrm{keV}$ neutrino mass squared differences these limits may overcome those that would apply in the case of zero magnetic field.
\end{abstract}

\section{Introduction}

Recently there has been ever growing hints for nonzero neutrino masses from solar and atmospheric neutrino observations $[1,2]$ as well as from the COBE data on cosmic background temperature anisotropies on large scales [3]. The latter indicate the need for the existence of a hot dark matter component, contributing about $30 \%$ of the total mass density [4]. Simple extensions of the standard electroweak model that can reconcile all these hints postulate the existence of a light sterile neutrino $\nu_{s}$ [5-7]. In some of these models such light sterile neutrino may play the role of hot dark matter [5,7]. Models with sterile neutrinos have also been suggested to account for the possible existence of $\mathrm{kcV}$ mass neutrinos [8]. Although the existing data do not support any positive claim, we stress that such neutrinos could well exist. Indeed the only limits on neutrino masses of general validity are those coming from direct laboratory searches. For example, the cosmological arguments that forbid neutrino masses in the $\mathrm{keV}$ range or above are not applicable in models with unstable neutrinos that decay via majoron emission [9].

So far the most stringent constraints for the neutrino mass matrix including a fourth neutrino species, $\nu_{s}$, are obtained from the nucleosynthesis bound on the maximum number of extra neutrino species $(\Delta N \leq 0.3)$ that can reach thermal equilibrium before nucleosynthesis and change the primordially produced helium abundance [10]. This has been widely discussed in the case of an isotropic early Universe hot plasma as well as recently for the case of a large random magnetic ficld that could arise from the clectrowcak phase transition and act as seed for the galactic magnetic fields [11].

\footnotetext{
${ }^{I}$ On leave from the Institute of the Terrestrial Magnetism, the Ionosphere and Radio Wave Propagation of the Russian Academy of Sciences, IZMIRAN, Troitsk, Moscow region, 142092, Russia.

${ }^{2}$ E-mail: VALLE at vm.ci.uv.es or 16444::VALLE
} 
In this letter we would like to consider the effect of a large random magnetic field in a supernova environment. The effect of active-sterile neutrino conversions in a supernova has been discussed in the case where no magnetic field is present. In this case stringent constraints can be placed on the $\nu_{a} \leftrightarrow \nu_{s}$ oscillation parameters. For the case $\Delta m^{2} \gtrsim \mathrm{keV}^{2}$ one can exclude the range [12]

$$
2 \times 10^{-2} \gtrsim \sin ^{2} 2 \theta \gtrsim 7 \times 10^{-10}
$$

In a recent paper [13] it was shown that magnetic fields as strong as $10^{14}$ to $10^{16}$ Gauss might be generated inside a supernova core due to a small scale dynamo mechanism during the first seconds of neutrino emission. If the magnetic field is generated after collapse this field could be viewed as the random superposition of many small dipoles of strength $10^{14}$ to $10^{16}$ gauss and size $L_{0} \sim 1 \mathrm{~km} \mathrm{[13].}$

In this paper we consider the effect of such huge random magnetic fields on the active sterile neutrino conversions. We show how such magnetic field can influence the neutrino spectrum in the supernova medium and thereby modify the neutrino conversion $\nu_{a} \rightarrow \nu_{s}$ rates. We shall confine ourselves to a given random magnetic field domain size $L_{0}$, within which the magnetic field may be taken as uniform and constant, so that the magnetization of the medium can be calculated easily. Although the magnetic field in different domains is randomly aligned relative to the neutrino propagation direction, the observable neutrino conversion probabilities depend on the mean-squared random field via a squared magnetization value, therefore leading to nonvanishing averages over the magnetic field distribution. We apply this to the active-sterile neutrino conversions in a supernova and show how their effect on the cooling rates may enable one to place limits that are more stringent than those that apply in the absence of a magnetic field.

Notice that the effect discussed in our present paper is more general than that which could also exist due to nonzero majorana neutrinos transition magnetic moments [14] as it would exist even if such magnetic moments are neglected.

\section{Magnetization effects on the active-sterile neutrino conversions in a supernova}

In this section we consider the active-sterile neutrino conversions in a supernova core in the presence of a strong random magnetic field. We will show here that in a strong magnetic field supernova constraints on neutrino oscillation parameters can be more stringent than in the case of zero magnetic field. The problem of active to sterile neutrino conversions in a supernova random magnetic field resembles the one considered in Ref. [11].

Starting from the general equations of motion for a system of one active $(S U(2) \otimes U(1)$ doublet) and one sterile $(S U(2) \otimes U(1)$ singlet) majorana neutrinos [15] one can write the evolution equation describing their propagation in the presence of a large random magnetic field, in terms of weak eigenstates, as

$$
i \frac{d}{d t}\left(\begin{array}{l}
\nu_{a} \\
\nu_{s}
\end{array}\right)=\left[\begin{array}{cc}
\left(c^{2} m_{1}^{2}+s^{2} m_{2}^{2}\right) / 2 q+V_{a s}+A_{a s} & c s \Delta \\
s c \Delta & \left(s^{2} m_{1}^{2}+c^{2} m_{2}^{2}\right) / 2 q
\end{array}\right]\left(\begin{array}{l}
\nu_{a} \\
\nu_{s}
\end{array}\right),
$$

where we have denoted by $V_{a s}$ the vector part of the neutrino potential that will describe the active to sterile conversions, given as

$$
V_{a s} \approx 4 \times 10^{-6} \rho_{14}\left(3 Y_{e}+4 Y_{\nu_{e}}-1\right) \mathrm{MeV} .
$$

For a magnetic field in the z-direction the corresponding axial part $A_{\mathrm{as}}(q, B)$ of the neutrino potential that will describe the active to sterile conversion is given by

$$
A_{\text {as }}(q, B)=V_{\text {axial }} \frac{q_{z}}{q}
$$


where the term $V_{\mathrm{axial}}=\mu_{\mathrm{eff}} B$ produced by the mean axial current is proportional to the magnetization of the plasma in the external magnetic field ${ }^{3}$ and quantity $\mu_{\text {eff }}$ is defined, for a degenerate electron gas, as

$$
\mu_{\mathrm{eff}}=\frac{e G_{F}\left(-2 c_{A}\right) p_{F_{e}}}{\sqrt{2} 2 \pi^{2}} \approx 4.3\left(-2 c_{A}\right) \times 10^{-13} \mu_{B}\left(\frac{p_{F_{e}}}{\mathrm{MeV}}\right) .
$$

Here $\mu_{B}=|e| / 2 m_{e}$ is the Bohr magneton, $c_{A}=\mp 0.5$ is the axial coupling specifying the interaction of charged leptons with neutrinos in the standard model (upper sign for $\nu_{e}$ and the lower holds for $\nu_{\mu}$ and $\nu_{\tau}$ ) and $p_{F_{e}}$ is the electron Fermi momentum, given as

$$
p_{r_{r}} \approx 320\left(Y_{e} \rho_{14}\right)^{1 / 3} \mathrm{MeV} .
$$

In the above equations $q$ is the neutrino momentum, $m_{1}$ and $m_{2}$ are the masses of the neutrinos, $\theta$ is their mixing angle and we use the standard definitions $\Delta=\Delta m^{2} / 2 q ; \Delta m^{2}=m_{2}^{2}-m_{1}^{2} ; c=\cos \theta$, and $s=\sin \theta$. In addition $\rho_{14}$ is the density of the stellar core in units $\rho=10^{14} \mathrm{~g} / \mathrm{cm}^{3}, Y_{e}$ and $Y_{\nu_{e}}$ are the electron and $\nu_{e}$ abundances respectively.

Notice that we have neglected the possible effect of nonzero transition magnetic moments. As we will see, even in this case, there may be a large effect of the magnetic field on the conversion rates.

From Eq. (2.1) one can easily obtain the probability $P_{\nu_{a} \rightarrow \nu_{s}}(t)$ for converting the active neutrinos $\nu_{a}$ emitted by the supernova into the sterile neutrinos, $\nu_{s}$. In a strong random magnetic field obeying the condition $\Gamma \gg \Delta_{m}$ one can write

$$
P_{\nu_{a} \rightarrow \nu_{s}}(B, t) \approx \frac{\Delta^{2} \sin ^{2} 2 \theta}{2 \Delta_{m}^{2}}\left(1-\exp \left(-\Delta_{m}^{2} t / 2 \Gamma\right)\right),
$$

which describes the aperiodic behaviour of the active to sterile neutrino conversion. The relaxation time defined as

$$
t_{\text {relax }}=2 \Gamma / \Delta_{m}^{2}=\left\langle\Delta_{B}^{2}\right\rangle L_{0} / \Delta_{m}^{2}
$$

depends on the mean squared magnetic field parameter

$$
\left\langle\Delta_{B}^{2}\right\rangle^{1 / 2}=\frac{\left|\mu_{\mathrm{eff}}\right|\left\langle\boldsymbol{B}^{2}\right\rangle^{1 / 2}}{\sqrt{3}},
$$

where $L_{0}$ is the domain size. In Eq. (2.6) the quantity $\Delta_{m}$

$$
\Delta_{m}=\left[\left(V_{\mathrm{as}}-\Delta \cos 2 \theta\right)^{2}+\Delta^{2} \sin ^{2} 2 \theta\right]^{1 / 2}
$$

is the standard oscillation frequency in the supernova medium [16].

In order to compare the relaxation time in Eq. (2.7) with the mean active neutrino collision time $t_{\text {coll }}=$ $\Gamma_{a}(B \neq 0)^{-1}$ we first write the active neutrino collision rate in the absence of magnetic field, obtained in Ref. [12] as

$$
\Gamma_{a}(B=0) \simeq \frac{7.5 \times G_{F}^{2}\langle E\rangle^{2}}{\pi} \times \frac{\rho}{m_{p}} .
$$

In the presence of a strong magnetic field $B \gg 2 B_{c}, B_{c}=m_{e}^{2} / e \approx 4.4 \times 10^{13}$ gauss, the neutrino collision rate may be estimated as [17],

\footnotetext{
${ }^{3}$ We assume this field to be uniform inside a given domain.
} 


$$
\Gamma_{a}(B \neq 0) \lesssim 2 B_{14} \Gamma_{a}(B=0)
$$

As we can see this collision rate could be larger than $\Gamma_{a}(B=0)$ by a factor $2 B_{14}$, where $B_{14}$ denotes the magnetic field strength in units of $10^{14}$ gauss.

It is easy to verify that the relaxation time Eq. (2.7) can be much larger than the mean active neutrino collision time $t_{\text {coll. }}$. This allows us, following Ref. [11], to average Eq. (2.6) over collisions so as to obtain

$$
\left\langle P_{\nu_{a} \rightarrow \nu_{s}}(B)\right\rangle=\frac{\Delta^{2} \sin ^{2} 2 \theta}{\left\langle\Delta_{B}^{2}\right\rangle 4 \Gamma_{a} L_{0}} .
$$

Now we come to the issue of the validity of our main formula, Eq. (2.6). For this we evaluate the damping parameter $\Gamma=\left\langle\Delta_{B}^{2}\right\rangle L_{0} / 2$ using Eq. (2.8) and Eq. (2.4). We find

$$
\Gamma \approx 5.3 \times 10^{-6}\left(Y_{e} \rho_{14}\right)^{2 / 3} B_{14}^{2} \mathrm{MeV} .
$$

Thus we see that the requirement that $\Gamma \gg \Delta_{m}$ is fulfilled for the case of a strong r.m.s. magnctic ficld obcying ${ }^{4}$

$$
B_{14} \gg 0.9 \times \rho_{14}^{1 / 6} \times \frac{\left(3 Y_{e}+4 Y_{\nu e}-1\right)^{1 / 2}}{Y_{e}^{1 / 3}} .
$$

It has been suggested that very large random magnetic fields as strong as $10^{14}-10^{16}$ gauss may in fact be generated inside a supernova core or a nascent neutron star due to a small scale dynamo mechanism that could take place after collapse during the first few seconds of neutrino emission [13]. These authors indeed suggested that such field could behave like a random superposition of many small dipoles of strength $\gtrsim 10^{14}$ gauss and size $L_{0} \sim 1 \mathrm{~km}$.

Note finally that in the opposite case of weak magnetic fields $\Gamma \rightarrow 0$ the probability Eq. (2.6) coincides with the averaged non-resonant MSW result [16]

$$
\left\langle P_{\nu_{u} \rightarrow \nu_{s}}(B \rightarrow 0)\right\rangle=\frac{\Delta^{2} \sin ^{2} 2 \theta}{2 \Delta_{m}^{2}} \equiv \frac{\sin ^{2} 2 \theta_{m}}{2}
$$

used in Ref. [12] in order to derive constraints on neutrino oscillation parameters in the case of zero magnetic field.

It will be convenient, in analogy to the case of zero magnetic field, $P_{\nu_{a} \rightarrow \nu_{s}}(B \rightarrow 0)=\sin ^{2} 2 \theta_{m} / 2$, to rewrite Eq. (2.12) as

$$
\left\langle P_{\nu_{a} \rightarrow \nu_{\mathrm{s}}}(B \neq 0)\right\rangle=\frac{\sin ^{2} 2 \theta_{B}}{2},
$$

where we define the mixing angle in the presence of the magnetic field via

$$
\sin ^{2} 2 \theta_{B}=\frac{\Delta^{2} \sin ^{2} 2 \theta}{2\left\langle\Delta_{B}^{2}\right\rangle \Gamma_{a} L_{0}}=\frac{x}{2} \sin ^{2} 2 \theta_{m} .
$$

We can define a parameter $x$ as

$$
x=\Delta_{m}^{2} / 2 \Gamma \Gamma_{a}(B \neq 0) .
$$

Using Eq. (2.11) we can write

\footnotetext{
${ }^{4}$ In this estimate we have assumed $\Delta_{m} \sim V_{\text {as. }}$.
} 


$$
x \gtrsim \frac{0.5 \times 10^{6} \rho_{14}^{1 / 3}\left(3 Y_{e}+4 Y_{\nu e}-1\right)^{2}}{B_{14}^{3} Y_{e}^{2 / 3}\left\langle E_{100}^{2}\right\rangle} .
$$

Notice that for a strong enough magnetic field, for instance, a r.m.s. field $B_{14} \sim 10^{2}$ the r.h.s. becomes less than unity if the abundance factor (for $\left\langle E_{100}^{2}\right\rangle=1$, i.e. for $100 \mathrm{MeV}$ neutrino energies) is less than

$$
\left|3 Y_{e}+4 Y_{\nu_{e}}-1\right| \leq Y_{e}^{1 / 3} \rho_{14}^{-1 / 6} .
$$

If, on the other hand, the argument in Eq. (2.18) is much less than unity, $x \ll 1$, the probability Eq. (2.6), averaged over fast collisions, reproduces the result in Eq. (2.12). Notice that the mixing angle in the presence of the magnetic field is smaller than with $B=0$, as the encrgy difference between the two diagonal entrics in Eq. (2.1) increases due to the presence of the extra axial term. Let us now turn to astrophysics.

\section{Supernova constraints}

There are two ways to place constraints on neutrino oscillation parameters using astrophysical criteria, depending on the relative value of the sterile neutrino effective mcan frce path $l_{s}=\Gamma_{s}^{-1}=\left[P\left(\nu_{a} \rightarrow \nu_{s}\right) \Gamma_{a}\right]^{-1}$ and the core radius $R_{\text {core }}$, where the effective sterile neutrino production rate $\Gamma_{s}$ is the product of the active neutrino production rate (which in our estimate we take to be the same as $\Gamma_{a}$ ) with the active to sterile conversion probability.

If the trapping condition $l_{s} \leq R_{\text {core }}$ is fulfilled, the $\nu_{\text {sterile }}$ are in thermodynamical equilibrium with the medium and, due to Stefan-Boltzman law, the ratio of the sterile neutrino luminosity to that of the ordinary neutrinos

$$
\frac{Q_{s}}{Q_{a}} \simeq\left(\frac{T\left(R_{s}\right)}{T\left(R_{a}\right)}\right)^{4}\left(\frac{R_{s}}{R_{a}}\right)^{2} \simeq\left(\frac{\Gamma_{a}}{\Gamma_{s}}\right)^{1 / 2}
$$

does not depend on $\Gamma_{a}$. In this first regime one considers surface thermal neutrino emission and sets the conservative limit $\left(Q_{s} / Q_{a}\right)_{\max } \gtrsim 10$ in order to obtain the excluded region of neutrino parameters. In the case of zero magnetic field one has

$$
\frac{Q_{s}}{Q_{a}} \simeq\left(\frac{\sin ^{2} 2 \theta_{m}}{2}\right)^{-1 / 2}
$$

which gives the excluded region [12]

$$
\sin ^{2} 2 \theta_{m} \lesssim 2 \times 10^{-2} \text {. }
$$

Another complementary constraint can be obtained from the requirement that in the non-trapping regime the sterile neutrino can be emitted from anywhere inside the star volume with a rate

$$
\frac{d Q(B=0)}{d t} \simeq \frac{4}{3} \pi R_{\text {core }}^{3} n_{\nu e} \Gamma_{s}\left\langle E_{s}\right\rangle \simeq 1.4 \times 10^{55} \sin ^{2} 2 \theta_{m} \frac{J}{S}
$$

which should not exceed the maximum observed integrated neutrino luminosity. For instance, for the case of SN1987A, this is $\sim 10^{46} \mathrm{~J}$. The corresponding collision rate should be less than $10^{46} \mathrm{~J} / \mathrm{s}$, so that one obtains the excluded region [12]

$$
\sin ^{2} 2 \theta_{m} \gtrsim 7 \times 10^{-10} \text {. }
$$

We now move to the case of strong magnetic field and how the constraints that can be obtained for this case compare with those of the case where the magnetic field is absent. For $B \neq 0$ we use the known estimate 


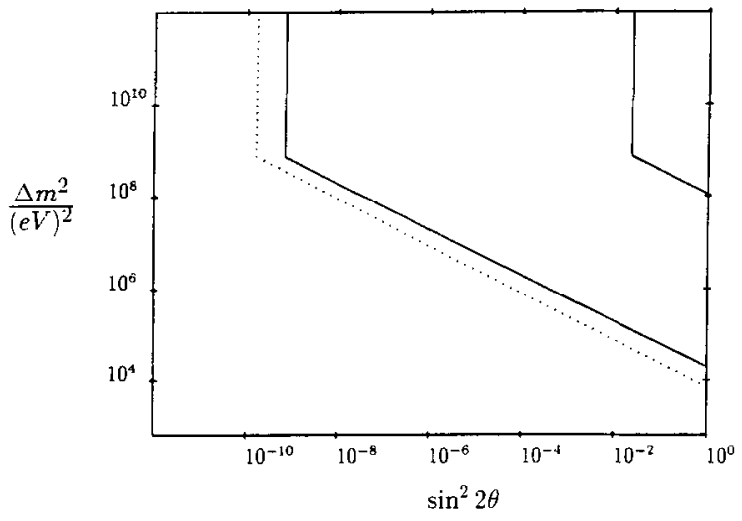

Fig. 3.1. Constraints on active to sterile neutrino conversions from a supernova with a strong random magnetic field. The region of $\nu_{e}$ to $\nu_{\text {sterile }} \mathrm{Sm}^{2} \sin ^{2} 2 \theta$ parameters above the dotted line is excluded by supernova cooling rates for aperiodic conversion in a strong random magnetic field $B=10^{16}$ gauss. Here we assume $Y_{e} \simeq 0.3, Y_{\nu e} \simeq 0.06$ and core density $\rho=8 \times 10^{14} \mathrm{~g} / \mathrm{cm}^{3}$. For comparison the region between the solid lines would be excluded for the same parameters for the case of $\nu_{a} \leftrightarrow \nu_{s}$ oscillations in the absence of magnetic field.

for the active neutrino collision rate $\Gamma_{a}(B \neq 0) \leq 2 B_{14} \Gamma_{a}(B=0)$ [17] and the relationship between the corresponding conversion probabilities in order to oblain $d Q(B \neq 0) / d t$. The ratio of sterile neutrino volume energy losses in the presence and absence of magnetic field may be easily obtained as

$$
\frac{d Q(B=0) / d t}{d Q(B \neq 0) / d t} \sim \frac{1}{x B_{14}}
$$

where $x$ is the small parameter in Eq. (2.18). From the last inequality we can find a region of abundances Eq. (2.19) where our result for the conversion probability Eq. $(2.12)$ is valid $(x \ll 1)$ so that we obtain the excluded region

$$
\sin ^{2} 2 \theta_{m} \gtrsim \frac{7 \times 10^{-10}}{x B_{14}}
$$

Note that this constraint on the neutrino parameters can be more stringent than that of Eq. (3.4).

In an analogous way we obtain the ratio of luminosities $Q_{s} / Q_{a}$ in the trapping regime (this is independent of the active neutrino collision ratc $\left.\Gamma_{a}(B)\right)$ and find that $\sin ^{2} 2 \theta_{B} \leq 2 \times 10^{-2}$ leading, from Eq. (2.16), to a new excluded region of the active-sterile neutrino mixing angle

$$
\sin ^{2} 2 \theta_{m} \lesssim \frac{4 \times 10^{-2}}{x}
$$

Note that this constraint on the neutrino parameters can be more stringent than that of Eq. (3.2). In particular, for a supernova with strong magnetic field it is possible to exclude all region of large mixing angles, if the parameter $x$ in Eq. (2.18) is $x \leq 0.04$. This will be realized for a r.m.s. field $B_{14} \sim 10^{2}[13]$ and mean sterile neutrino energy $\left\langle E_{100}^{2}\right\rangle=1$ if the abundance parameter is less than

$$
\left|3 Y_{e}+4 Y_{\nu e}-1\right| \leq 0.3 \times Y_{e}^{1 / 3} \rho_{14}^{-1 / 6} .
$$

This condition can indeed be realized for a stage of supernova after bounce [12,18]. Moreover, this assumption is not crucial for us, in contrast to the case of resonant neutrino spin-flip due to a neutrino magnetic moment considered in Ref. [19].

We now summarize this discussion with one example. Our goal is to display the above constraints in terms of the parameters $\Delta m^{2} \sin ^{2} 2 \theta$. In order to do this we use Eq. (2.15) to relate the neutrino parameters in matter 
with those in vacuum. In Fig. 1 we show the constraints on neutrino parameters that follow from supernova cooling rates in the presence of a strong random magnetic field. The region of $\nu_{e}$ to $\nu_{\text {sterile }}$ parameters ${ }^{5}$ above the dotted line is excluded by supernova cooling rates for aperiodic conversion in a random magnetic field $B=10^{16}$ gauss. In contrast, in the case of zero magnetic field, for the same parameters only the points between the solid lines would be excluded.

\section{Discussion and conclusions}

The possible existence of huge random magnetic fields that might generated during the first few seconds of neutrino emission in a supernova modifies the neutrino spectrum due to the magnetization of the medium.

Averaging the differential equation describing the evolution of the $\nu_{a} \rightarrow \nu_{s}$ conversion probabilities over the random magnetic field distribution we find a nonvanishing mean squared field effect which may drastically change the conversion rates with respect to those which hold in the case of zero magnetic field. This may be used in order to derive new and potentially more stringent constraints on the active-sterile neutrino oscillation parameters than in the case without random magnetic field. This happens despite the fact that in the presence of a large magnetic field the active to sterile neutrino conversion probability is suppressed relative to that in the zero magnetic field case due to the larger energy difference between the two diagonal entries in the neutrino evolution hamiltonian Eq. (2.1) caused by the presence of the extra axial term. This happens because the sterile neutrino production rate could be larger in this case due to effect of the large magnetic field.

On the other hand the ratio of active and sterile neutrino thermal luminosities does not depend on the active neutrino production rate. However, the smaller the conversion probability the larger the sterile neutrino effective mean free path and therefore they can leave the star more easily than in the case of zero magnetic field. This leads to the possible exclusion of the complete large mixing angle region, as illustrated in Fig. 1.

Notice that, although majorana neutrinos could have nonzero transition magnetic moments [14], we have neglected them in our present discussion. As we have seen, even in this case, there may be a large effect of the magnetic field on the neutrino conversion rates. The effect which we have found is therefore of more general validity than that which could be ascribed to nonzero electromagnetic moments. The latter are expected to be small in the simplest extensions of the standard model.

\section{Acknowledgements}

This work was supported by DGICYT under grant number PB92-0084 and partially by the EEC under grant number CHRX-CT93-0132, and by a senior researcher NATO fellowship (V.S.). We thank Armando Perez for pointing out to us the paper in Ref. [13] and also Juan Antonio Miralles for fruitful discussions.

\section{References}

[1] T. Kirsten, Phys. Lett. B 314 (1993) 445; Kamiokande, SAGE, GALLEX collaboration talks at XV Int. Conference on Neutrino Physics and Astrophysics, Nucl. Phys. B (Poc. Suppl.) 31 (1993) 105-124; J.R. Davis in Proceedings of the 21th International Cosmic Ray Conference, Vol. 12, ed. R.J. Protheroe (University of Adelaide Press, 1990) p. 293.

[2] Kamiokande collaboration, Phys. Lett. B 205 (1988) 416, Phys. Lett. B 280 (1992) 146 and Phys. Lett. B 283 (1992) 446; IMB collaboration, Phys. Rev. D 46 (1992) 3720; see also the proceedings of Int. Workshop on $\nu_{\mu} / \nu_{e}$ problem in atmospheric neutrinos ed. V. Berezinsky and G Fiorentini, Gran Sasso, 1993.

[3] G.F. Smoot et al., Astrophys. J. 396 (1992) L1-L5.

\footnotetext{
${ }^{5}$ Similarly one may derive constraints on $\nu_{\mu}$ or $\nu_{\tau}$ to $\nu_{\text {sterile }}$ conversions.
} 
[4] E.L. Wright et al., Astrophys. J. 396 (1992) L13; M. Davis, F.J. Summers, and D. Schagel, Nature 359 (1992) 393; A.N. Taylor and M. Rowan-Robinson, ibid 359 (1992) 396; R.K. Schaefer and Q. Shafi, Nature 359 (1992) 199; J.A. Holtzman and J.R. Primack, Astrophys. J. 405 (1993) 428; A. Klypin et al., Astrophys. J. 416 (1993) 1.

[5] J. T. Peltoniemi, D. Tommasini, and J. W. F. Valle, Phys. Lett. B 298 (1993) 383.

[6] J.T. Peltoniemi, and J.W.F. Valle, Nucl. Phys. B 406 (1993) 409; D.O. Caldwell and R.N. Mohapatra, Phys. Rev. D 48 (1993) 3259.

[7] E. Akhmedov, Z. Berezhiani, G. Senjanovic and Z. Tao, Phys. Rev. D 47 (1993) 3245; J.T. Peltoniemi, Mod. Phys. Lett. A 38 (1993) 3593.

[8] L. Bento and J.W.F.Valle, Phys. Lett. B 264 (1991) 373; A. Smimov and J.W.F.Valle, Nucl. Phys. B 375 (1992) 649; J. Peltoniemi, A. Smirnov and J.W.F.Valle, Phys. Lett. B 286 (1992) 321.

[9] For a recent review see J.W.F. Valle, in Gauge Theories and the Physics of Neutrino Mass, Prog. Part. Nucl. Phys. 26 (1991) $91-171$ and references therein.

[ 101 T.Walker, G.Steigman, D.N.Schramm, K.Olive, and H.Kang, Astrophys. J. 376 (1991) 51.

[11] V. Semikoz and J.W.F.Valle, Valencia Preprint FTUV/94-05, to be published in Nucl. Phys. B (1994) .

[12] K. Kainulainen, J. Maalampi and J.T. Peltoniemi, Nucl. Phys. B 358 (1991) 435; G. Raffelt and G. Sigl, Astroparticle Physics 1 (1993) 165

[13] C. Thomson and R.C. Dunkan, Astrophys. J. 408 (1993) 194.

[14] J. Schechter and J. W. F. Valle, Phys. Rev, D 24 (1981) 1883; Phys. Rev. D 25 (1982) 283.

[15] S. Pastor, V. Semikoz and J.W.F.Valle, in preparation.

[16] M. Mikheyev, A. Smirnov, Sov. J. Nucl. Phys. 42 (1986) 913; L. Wolfenstein, Phys. Rev. D 17 (1978) 2369 ;ibid D 20 (1979) 2634.

[17] B. Cheng, D.N. Schramm and J.W. Truran, Phys. Lett. B 316 (1993) 521.

[18] D. Notzold Phys. Rev. D 38 (1988) 1658.

[19] M.B. Voloshin, Phys. Lett. B 209 (1988) 360. 